\title{
Marcinkiewicz Integrals on Weighted Weak Hardy Spaces
}

\author{
Yue $\mathrm{Hu}^{1}$ and Yueshan Wang ${ }^{2}$ \\ ${ }^{1}$ College of Mathematics and Informatics, Henan Polytechnic University, Jiaozuo 454003, China \\ ${ }^{2}$ Department of Mathematics, Jiaozuo University, Jiaozuo 454003, China
}

Correspondence should be addressed to Yue Hu; huu3y3@163.com

Received 13 January 2014; Accepted 26 February 2014; Published 3 April 2014

Academic Editor: Yongqiang Fu

Copyright (C) 2014 Y. Hu and Y. Wang. This is an open access article distributed under the Creative Commons Attribution License, which permits unrestricted use, distribution, and reproduction in any medium, provided the original work is properly cited.

We prove that, under the condition $\Omega \in \operatorname{Lip}_{\alpha}$, Marcinkiewicz integral $\mu_{\Omega}$ is bounded from weighted weak Hardy space $\mathrm{WH}_{w}^{p}\left(R^{n}\right)$ to weighted weak Lebesgue space $\mathrm{WL}_{w}^{p}\left(R^{n}\right)$ for $\max \{n /(n+1 / 2), n /(n+\alpha)\}<p \leq 1$, where $w$ belongs to the Muckenhoupt weight class. We also give weaker smoothness condition assumed on $\Omega$ to imply the boundedness of $\mu_{\Omega}$ from $\mathrm{WH}_{w}^{1}\left(\mathbb{R}^{n}\right)$ to $\mathrm{WL}_{w}^{1}\left(R^{n}\right)$.

\section{Introduction and Results}

Suppose that $S^{n-1}$ is the unit sphere in $R^{n}(n \geq 2)$ equipped with Lebesgue measure $d \sigma\left(x^{\prime}\right)$. Let $\Omega$ be homogeneous of degree zero on $R^{n}$ satisfying $\Omega \in L^{1}\left(S^{n-1}\right)$ and

$$
\int_{S^{n-1}} \Omega\left(x^{\prime}\right) d \sigma\left(x^{\prime}\right)=0,
$$

where $x^{\prime}=x /|x|$ for any $x \neq 0$. Then, the Marcinkiewicz integral operator $\mu_{\Omega}$ is defined by

$$
\mu_{\Omega}(f)(x)=\left(\int_{0}^{\infty}\left|F_{\Omega, t}(f)(x)\right|^{2} \frac{d t}{t^{3}}\right)^{1 / 2}
$$

where

$$
F_{\Omega, t}(f)(x)=\int_{|x-y| \leq t} \frac{\Omega(x-y)}{|x-y|^{n-1}} f(y) d y .
$$

The operator $\mu_{\Omega}$ was originally introduced by Marcinkiewicz [1] in 1938 for $n=1$ and $\Omega(t)=\operatorname{sign} t$. In 1958, Stein [2] defined the Marcinkiewicz integral of higher dimensions and proved that if $\Omega \in \operatorname{Lip}_{\alpha}\left(S^{n-1}\right), 0<\alpha \leq 1$, then $\mu_{\Omega}$ is of strong type $(p, p)$ for $1<p \leq 2$ and of weak type $(1,1)$. We say that $\Omega \in \operatorname{Lip}_{\alpha}\left(S^{n-1}\right)$ if

$$
\left|\Omega\left(x^{\prime}\right)-\Omega\left(y^{\prime}\right)\right| \leq\left|x^{\prime}-y^{\prime}\right|^{\alpha}, \quad x^{\prime}, y^{\prime} \in S^{n-1} .
$$

In 1962, Benedek et al. [3] showed that if $\Omega$ is continuously differentiable on $S^{n-1}$, then $\mu_{\Omega}$ is of strong type $(p, p)$ for all $1<p<\infty$. In 1990, Torchinsky and Wang [4] considered the weighted case and proved that if $\Omega \in \operatorname{Lip}_{\alpha}\left(S^{n-1}\right), 0<\alpha \leq$ 1 , then, for all $w \in A_{p}$ (Muckenhoupt weight class), $\mu_{\Omega}$ is bounded on $L_{w}^{p}\left(R^{n}\right)$.

In 1999, Ding et al. [5] improved Torchinsky and Wang's result, by ridding $\Omega$ of the smoothness condition, as follows.

Theorem A. Let $\Omega \in L^{q}\left(S^{n-1}\right), 1<q \leq \infty$, and satisfy (1). If $w^{q^{\prime}} \in A_{p}, 1<p<\infty$, then there is $C>0$ independent of $f$ such that

$$
\left\|\mu_{\Omega}(f)\right\|_{L_{w}^{p}} \leq C\|f\|_{L_{w}^{p}}
$$

where $q^{\prime}$ is the dual of $q$ such that $1 / q+1 / q^{\prime}=1$.

In 2003, Ding et al. [6] discussed the boundedness of Marcinkiewicz integral on weighted Hardy space, getting the following results.

Theorem B. Let $0<\alpha \leq 1, \beta=\min \{\alpha, 1 / 2\}$, and $n /(n+$ $\beta)<p<1$. Let $\Omega \in \operatorname{Lip}_{\alpha}\left(R^{n}\right)$ satisfy (1). If $w \in A_{p(1+\beta / n)}$, then there exists a constant $C>0$ independent of $f$ such that $\left\|\mu_{\Omega}(f)\right\|_{L_{w}^{p}} \leq C\|f\|_{H_{w}^{p}}$.

Theorem C. Let $\Omega$ satisfy (1) and $L^{q}$ condition $(q>1)$. If $w^{q^{\prime}} \in A_{1}$, then there is a $C>0$ independent of $f$ such that $\left\|\mu_{\Omega}(f)\right\|_{L_{w}^{1}} \leq C\|f\|_{H_{w}^{1}}$. 
In 2007, C. C. Lin and Y. C. Lin [7] showed that under weaker condition assumed on $\Omega$, which is called $L^{q}$-Dini type condition of order $\alpha$, Marcinkiewicz integral operator $\mu_{\Omega}$ is bounded from $H_{w}^{p}$ to $L_{w}^{p}$.

We say that $\Omega$ satisfies the $L^{q}$-Dini condition if $\Omega \in$ $L^{q}\left(S^{n-1}\right), q \geq 1$, is homogenous of degree zero on $R^{n}$, and

$$
\int_{0}^{\infty} \frac{w_{q}(\delta)}{\delta} d \delta<\infty
$$

where $w_{q}(\delta)$ denotes the integral modulus of continuity $q$ of $\Omega$ defined by

$$
w_{q}(\delta)=\sup _{|\rho|<\delta}\left(\int_{S^{n-1}}\left|\Omega\left(\rho x^{\prime}\right)-\Omega\left(x^{\prime}\right)\right|^{q} d \sigma\left(x^{\prime}\right)\right)^{1 / q},
$$

and $\rho$ is a rotation in $R^{n}$ with $|\rho|=\|\rho-I\|$. If $q=1$, we let $w(\delta)=w_{q}(\delta)$.

In 2002, Ding et al. [8] considered the boundedness of Marcinkiewicz integral on weak Hardy space; they obtained the following result.

Theorem D. Suppose that $\Omega \in L^{1}\left(S^{n-1}\right)$ satisfies (1) and

$$
\int_{0}^{1} \frac{w(\delta)}{\delta}(1+|\log \delta|)^{\sigma} d \delta<\infty, \quad \text { for some } \sigma>1
$$

Then, there exists a constant $C>0$ such that

$$
\left\|\mu_{\Omega}(f)\right\|_{W L^{1}} \leq C\|f\|_{W H^{1}} \text {. }
$$

In this paper, we will investigate the boundedness of Marcinkiewicz integral on weighted weak Hardy space. We have the following conclusions.

Theorem 1. Suppose that $\Omega \in \operatorname{Lip}_{\alpha}\left(R^{n}\right), 0<\alpha \leq 1$, satisfies (1) and $\beta=\min \{\alpha, 1 / 2\}$. If $n /(n+\beta)<p \leq 1$ and $w \epsilon$ $A_{p(1+\beta / n)}$, then there exists a constant $C>0$ independent of $f$ such that $\left\|\mu_{\Omega}(f)\right\|_{W L_{w}^{p}} \leq C\|f\|_{W H_{w}^{p}}$.

Theorem 2. Let $\Omega \in L^{q}\left(S^{n-1}\right), 1<q \leq \infty$, satisfy (1) and

$$
\int_{0}^{1} \frac{w_{q}(\delta)}{\delta}(1+|\log \delta|)^{\sigma} d \delta<\infty, \quad \text { for some } \sigma>1
$$

If $w^{q^{\prime}} \in A_{1}$, then there is a $C>0$ independent of $f$ such that $\left\|\mu_{\Omega}(f)\right\|_{W L_{w}^{1}} \leq C\|f\|_{W H_{w}^{1}}$.

Remark 3. From [8, Remark 2], Condition (10) is weaker than Condition (4).

\section{Some Preliminaries and Notations}

We begin this section with some properties of $A_{p}$ weights which play an important role in the proofs of our main results.

A weight $w$ is a nonnegative, locally integrable function on $R^{n}$. Let $B=B\left(x_{0}, r_{B}\right)$ denote the ball with the center $x_{0}$ and radius $r_{B}$. For any ball $B$ and $\lambda>0, \lambda B$ denotes the ball concentric with $B$ whose radius is $\lambda$ times as long. For a given weight function $w$ and a measurable set $E$, we also denote the Lebesgue measure of $E$ by $|E|$ and set weighted measure $w(E)=\int_{E} w(x) d x$.
Definition 4. A weight $w$ is said to belong to $A_{p}$ for $1<p<$ $\infty$, if there exists a constant $C$ such that for every ball, $B \subset R^{n}$,

$$
\left(\frac{1}{|B|} \int_{B} w(x) d x\right)\left(\frac{1}{|B|} \int_{B} w(x)^{1-p^{\prime}} d x\right)^{p-1} \leq C .
$$

The class $A_{1}$ is defined by replacing the above inequality with

$$
\begin{array}{r}
\frac{1}{|B|} \int_{B} w(y) d y \leq C \cdot \text { ess } \inf _{x \in B} w(x) \\
\text { for every ball } B \subseteq R^{n} .
\end{array}
$$

The classical $A_{p}$ weight theory was first introduced by Muckenhoupt in the study of weighted $L^{p}$ boundedness of Hardy-Littlewood maximal functions in [9].

We know that if $w \in A_{p}$ with $1<p<\infty$, then $w \in A_{r}$ for all $r>p$ and $w \in A_{q}$ for some $1<q<p$. Therefore, we will use the notation $q_{w} \equiv \inf \left\{q>1: w \in A_{q}\right\}$ to denote the critical index of $w$. Obviously, if $w \in A_{q}, q>1$, then we have $1 \leq q_{w}<q$.

We state the following results about $A_{p}$ class that will be used later on.

Lemma 5 (see [10]). Let $w \in A_{p}, p \geq 1$. Then, for any ball $B$ and any $\lambda>1$, there exists an absolute constant $C>0$ such that

$$
w(\lambda B) \leq C \lambda^{n p} w(B)
$$

where $C$ depends neither on $B$ nor on $\lambda$.

Lemma 6 (see [10]). Let $w \in A_{q}$ with $q \geq 1$. Then, for any $r>0$, there exists an absolute $C>1$ independent of $r$ such that

$$
\int_{\left|x-x_{0}\right| \geq r} \frac{w(x)}{\left|x-x_{0}\right|^{n q}} d x \leq C r^{-n q} w\left(B\left(x_{0}, 2 r\right)\right) .
$$

We also need the following result about $L^{q}$-Dini condition.

Lemma 7 (see [11]). Suppose that $q>1$ and $\Omega$ satisfies $L^{q}$ Dini condition. Then, for $|y| \leq R / 2$,

$$
\begin{gathered}
\left(\int_{R<|x|<2 R}\left|\frac{\Omega(x-y)}{|x-y|^{n-1}}-\frac{\Omega(x)}{|x|^{n-1}}\right|^{q} d x\right)^{1 / q} \\
\leq C R^{n / q-n+1}\left(\frac{|y|}{R}+\int_{|y| / 2 R<\delta<|y| / R} \frac{w_{q}(\delta)}{\delta} d \delta\right) .
\end{gathered}
$$

Given a Muckenhoupt weight function $w$ on $R^{n}$, for $0<$ $p<\infty$, we denote by $L_{w}^{p}$ the space of all functions satisfying

$$
\|f\|_{L_{w}^{p}}=\left(\int_{R^{n}}|f(x)|^{p} w(x) d x\right)^{1 / p}<\infty .
$$

When $q=\infty, L_{w}^{\infty}$ will be taken to mean $L^{\infty}$, and we set $\|f\|_{L_{w}^{\infty}}=\|f\|_{L^{\infty}}$. And also the weighted weak Lebesgue spaces is defined by

$$
\|f\|_{\mathrm{WL}_{w}^{p}}=\sup _{\lambda>0} \lambda w\left(\left\{x \in R^{n}:|f(x)|>\lambda\right\}\right)^{1 / p}<\infty .
$$


Let us now turn to the weighted weak Hardy spaces. The (unweighted) weak $H^{p}$ spaces have first appeared in the work of Fefferman et al. [12]. The atomic decomposition theory of weak $H^{1}$ space on $R^{n}$ was given by Fefferman and Soria in [13]. In 2000, Quek and Yang [14] introduced the weighted weak Hardy spaces $\mathrm{WH}_{w}^{p}$ and established their atomic decompositions. Moreover, by using the atomic decomposition theory of $\mathrm{WH}_{w}^{p}$, Quek and Yang also obtained the boundedness of Calderón-Zygmund type operators on these weighted spaces.

We write $\delta\left(R^{n}\right)$ to denote the Schwartz space of all rapidly decreasing smooth functions and $\mathcal{S}^{\prime}\left(R^{n}\right)$ to denote the space of all tempered distributions, that is, the topological dual of $\mathcal{S}\left(R^{n}\right)$. Let $w \in A_{\infty}, 0<p \leq 1$, and $N=\left[n\left(q_{w} / p-1\right)\right]$. Define

$$
\begin{aligned}
\mathscr{A}_{N, w}= & \left\{\varphi \in \mathcal{S}\left(R^{n}\right): \sup _{x \in R^{n}|\alpha| \leq N+1} \sup _{\mid}(1+|x|)^{N+n+1}\right. \\
& \left.\times\left|D^{\alpha} \varphi(x)\right| \leq 1\right\}
\end{aligned}
$$

where $\alpha=\left(\alpha_{1}, \ldots, \alpha_{n}\right) \in(\mathbb{N} \cup\{0\})^{n},|\alpha|=\alpha_{1}+\cdots+\alpha_{n}$, and $D^{\alpha} \varphi=\partial^{|\alpha|} \varphi /\left(\partial x_{1}^{\alpha_{1}}, \ldots, \partial x_{n}^{\alpha_{n}}\right)$.

For $\mathcal{S}^{\prime}\left(R^{n}\right)$ the grand maximal function of $f$ is defined by

$$
G_{w}(f)(x)=\sup _{\varphi \in \mathscr{A}_{N, w}} \sup _{|x-y|<t}\left|\left(\varphi_{t} * f\right)(y)\right| .
$$

Then, we can define the weighted weak Hardy space $\mathrm{WH}_{w}^{p}\left(R^{n}\right)$ by

$$
\mathrm{WH}_{w}^{p}\left(R^{n}\right)=\left\{\mathcal{S}^{\prime}\left(R^{n}\right): G_{w} f \in \mathrm{WL}_{w}^{p}\left(R^{n}\right)\right\} .
$$

Moreover, we set $\|f\|_{\mathrm{WH}_{w}^{p}}=\left\|G_{w} f\right\|_{\mathrm{WL}_{w}^{p}}$.

Lemma 8 (see [14]). Let $0<p \leq 1$ and $w \in A_{\infty}$. For every $f$ in $W H_{w}^{p}\left(R^{n}\right)$, there exists a sequence of bounded measurable functions $\left\{f_{k}\right\}_{k=-\infty}^{\infty}$ such that

(i) $f=\sum_{k=-\infty}^{\infty} f_{k}$ in the sense of distributions.

(ii) Each $f_{k}$ can be further decomposed into $f_{k}=\sum_{i} b_{i}^{k}$, where $\left\{b_{i}^{k}\right\}$ satisfies the following:

(a) each $b_{i}^{k}$ is supported in a ball $B_{i}^{k}$ with $\sum_{i} w\left(B_{i}^{k}\right) \leq$ $c 2^{-k p}$ and $\sum_{i} \chi_{B_{i}^{k}}(x) \leq c$. Here, $\chi_{E}$ denotes the characteristic function of the set $E$ and $c \sim$ $\|f\|_{W H_{w}^{p}}$;

(b) $\left\|b_{i}^{k}\right\|_{L^{\infty}} \leq C 2^{k}$, where $C>0$ is independent of $i$ and $k$;

(c) $\int_{R^{n}} b_{i}^{k}(x) x^{\alpha} d x=0$ for every multi-index $\alpha$ with $|\alpha| \leq\left[n\left(q_{w} / p-1\right)\right]$.

Conversely, if $f \in \mathcal{S}^{\prime}\left(R^{n}\right)$ has a decomposition satisfying (i) and (ii), then $f \in W H_{w}^{p}\left(R^{n}\right)$. Moreover, we have $\|f\|_{W H_{w}^{p}} \sim c$.

\section{Proof of Theorem 1}

It suffices to show that there exists a constant $C>0$, such that, for any $f \in \mathrm{WH}_{w}^{p}$ and $\beta>0$,

$$
\beta^{p} w\left(\left\{x \in R^{n}:\left|\mu_{\Omega}(f)(x)\right|>\beta\right\}\right) \leq C\|f\|_{\mathrm{WH}_{w}^{p}}^{p} .
$$

For any given $\beta>0$, we choose $k_{0} \in \mathbb{Z}$ such that $2^{k_{0}} \leq$ $\beta<2^{k_{0}+1}$. Then, by Lemma 8 , for every $f \in \mathrm{WH}_{w}^{p}$, we can write

$$
f=\sum_{k=-\infty}^{\infty} f_{k}=\sum_{k=-\infty}^{k_{0}} f_{k}+\sum_{k=k_{0}+1}^{\infty} f_{k}=F_{1}+F_{2},
$$

where

$$
F_{1}=\sum_{k=-\infty}^{k_{0}} f_{k}=\sum_{k=-\infty}^{k_{0}} \sum_{i} b_{i}^{k}, \quad F_{2}=\sum_{k=k_{0}+1}^{\infty} f_{k}=\sum_{k=k_{0}+2}^{\infty} \sum_{i} b_{i}^{k}
$$

and $\left\{b_{i}^{k}\right\}$ satisfies (a)-(c) in Lemma 8. Then,

$$
\begin{aligned}
\beta^{p} & \left(\left\{x \in R^{n}:\left|\mu_{\Omega}(f)(x)\right|>\beta\right\}\right) \\
\leq & \beta^{p} w\left(\left\{x \in R^{n}:\left|\mu_{\Omega}\left(F_{1}\right)(x)\right|>\frac{\beta}{2}\right\}\right) \\
& +\beta^{p} w\left(\left\{x \in R^{n}:\left|\mu_{\Omega}\left(F_{2}\right)(x)\right|>\frac{\beta}{2}\right\}\right) \\
= & M_{1}+M_{2} .
\end{aligned}
$$

We claim that the following inequality holds:

$$
\left\|F_{1}\right\|_{L_{w}^{2}} \leq C \beta^{1-p / 2}\|f\|_{\mathrm{WH}_{w}^{p}}^{p / 2} .
$$

In fact, it follows from Minkowski's integral inequality and the bounded overlapping property of the cubes $\left\{B_{i}^{k}\right\}$ that

$$
\begin{aligned}
\left\|F_{1}\right\|_{L_{w}^{2}} & \leq \sum_{k=-\infty}^{k_{0}} \sum_{i}\left\|b_{i}^{k}\right\|_{L_{w}^{2}} \\
& \leq \sum_{k=-\infty}^{k_{0}} \sum_{i}\left\|b_{i}^{k}\right\|_{L^{\infty}} w\left(B_{i}^{k}\right)^{1 / 2} \\
& \leq C \sum_{k=-\infty}^{k_{0}} 2^{k}\left(\sum_{i} w\left(B_{i}^{k}\right)\right)^{1 / 2} \\
& \leq C \sum_{k=-\infty}^{k_{0}} 2^{k(1-p / 2)}\|f\|_{\mathrm{WH}_{w}^{p}}^{p / 2} \\
& \leq C \sum_{k=-\infty}^{k_{0}} 2^{\left(k-k_{0}\right)(1-p / 2)} \beta^{1-p / 2}\|f\|_{\mathrm{WH}_{w}^{p / 2}}^{p} \\
& \leq C \beta^{1-p / 2}\|f\|_{\mathrm{WH}_{w}^{p / 2}}^{p .}
\end{aligned}
$$


Notice that $w \in A_{p(1+\beta / n)} \subset A_{2}$; then from Chebyshev's inequality and the boundedness of $\mu_{\Omega}$ on $L_{w}^{2}$ we have

$$
M_{1} \leq \beta^{p} \frac{C}{\beta^{2}}\left\|\mu_{\Omega}\left(F_{1}\right)\right\|_{L_{w}^{2}}^{2} \leq C\|f\|_{\mathrm{WH}_{w}^{p}}^{p}
$$

We set

$$
A_{k_{0}}=\bigcup_{k=k_{0}+1}^{\infty} \bigcup_{i} \widetilde{B_{i}^{k}}
$$

where $\widetilde{B_{i}^{k}}=B\left(x_{i}^{k},(3 / 2)^{\left(k-k_{0}\right) /(n+\beta)} \sqrt{n} d_{i}^{k}\right)$. Then, we get

$$
\begin{aligned}
M_{2} \leq & \beta^{p} w\left(\left\{x \in A_{k_{0}}:\left|\mu_{\Omega}\left(F_{2}\right)(x)\right|>\frac{\beta}{2}\right\}\right) \\
& +\beta^{p} w\left(\left\{x \in\left(A_{k_{0}}\right)^{c}:\left|\mu_{\Omega}\left(F_{2}\right)(x)\right|>\frac{\beta}{2}\right\}\right) \\
= & M_{21}+M_{22} .
\end{aligned}
$$

Since $w \in A_{p(1+\beta / n)}$, then, by Lemmas 5 and 8 and the fact $2^{k_{0}} \leq \beta<2^{k_{0}+1}$, we get

$$
\begin{aligned}
M_{21} & \leq \beta^{p} w\left(A_{k_{0}}\right) \\
& \leq \beta^{p} \sum_{k=k_{0}+2}^{\infty} \sum_{i} w\left(\widetilde{B_{i}^{k}}\right) \\
& \leq C \beta^{p} \sum_{k=k_{0}+2}^{\infty}\left(\frac{3}{2}\right)^{\left(k-k_{0}\right) p} \sum_{i} w\left(B_{i}^{k}\right) \\
& \leq C\|f\|_{\mathrm{WH}_{w}^{p}}^{p} \sum_{k=k_{0}+2}^{\infty}\left(\frac{3}{4}\right)^{\left(k-k_{0}\right) p} \\
& \leq C\|f\|_{\mathrm{WH}_{w}^{p}}^{p} .
\end{aligned}
$$

An application of Chebyshev's inequality gives us that

$$
\begin{aligned}
M_{22} & \leq C \int_{\left(A_{k_{0}}\right)^{c}}\left|\mu_{\Omega}\left(F_{2}\right)\right|^{p} w(x) d x \\
& \leq C \sum_{k=k_{0}+1}^{\infty} \sum_{i} \int_{\left(\widetilde{B_{i}^{k}}\right)^{c}}\left|\mu_{\Omega}\left(b_{i}^{k}\right)(x)\right|^{p} w(x) d x .
\end{aligned}
$$

Below, we will give the estimate of integral $I=$ $\int_{\left(\bar{B}_{i}^{k}\right)^{c}}\left|\mu_{\Omega}\left(b_{i}^{k}\right)(x)\right|^{p} w(x) d x$. We have

$$
\begin{aligned}
I \leq \int_{\left(\bar{B}_{i}^{k}\right)^{c}} & \left(\int_{0}^{\left|x-x_{i}^{k}\right|+\sqrt{n} d_{i}^{k}}\left|\int_{|x-y| \leq t} \frac{\Omega(x-y)}{|x-y|^{n-1}} b_{i}^{k}(y) d y\right|^{2} \frac{d t}{t^{3}}\right)^{p / 2} \\
& \times w(x) d x \\
& +\int_{\left(\bar{B}_{i}^{k}\right)^{c}}\left(\int_{\left|x-x_{i}^{k}\right|+\sqrt{n} d_{i}^{k}}^{\infty}\left|\int_{|x-y| \leq t} \frac{\Omega(x-y)}{|x-y|^{n-1}} b_{i}^{k}(y) d y\right|^{2} \frac{d t}{t^{3}}\right)^{p / 2} \\
& \times w(x) d x \\
= & I_{1}+I_{2} .
\end{aligned}
$$

For $y \in B_{i}^{k}$ and $x \in\left(\widetilde{B_{i}^{k}}\right)^{c}$, we have $|x-y| \approx\left|x-x_{i}^{k}\right| \approx$ $\left|x-x_{i}^{k}\right|+\sqrt{n} d_{i}^{k}$. Thus,

$$
\left|\frac{1}{|x-y|^{2}}-\frac{1}{\left(\left|x-x_{i}^{k}\right|+\sqrt{n} d_{i}^{k}\right)^{2}}\right| \leq \frac{C d_{i}^{k}}{|x-y|^{3}} .
$$

By Minkowski’s inequality for integral,

$$
\begin{aligned}
& \left(\int_{0}^{\left|x-x_{i}^{k}\right|+\sqrt{n} d_{i}^{k}}\left|\int_{|x-y| \leq t} \frac{\Omega(x-y)}{|x-y|^{n-1}} b_{i}^{k}(y) d y\right|^{2} \frac{d t}{t^{3}}\right)^{1 / 2} \\
& \quad \leq \int_{B_{i}^{k}} \frac{|\Omega(x-y)|}{|x-y|^{n-1}}\left|b_{i}^{k}(y)\right|\left(\int_{|x-y|}^{\left|x-x_{i}^{k}\right|+\sqrt{n} d_{i}^{k}} \frac{d t}{t^{3}}\right)^{1 / 2} d y \\
& \quad \leq \frac{C 2^{k}\left(d_{i}^{k}\right)^{n+1 / 2}}{\left|x-x_{i}^{k}\right|^{n+1 / 2}} .
\end{aligned}
$$

From the fact that $q=(1+\beta / n) p \leq(1+1 / 2 n) p$ and Lemma 6 , we have

$$
\begin{aligned}
I_{1} \leq & C 2^{k p}\left(d_{i}^{k}\right)^{(n+1 / 2) p} \int_{\left(\widetilde{B_{i}^{k}}\right)} \frac{w(x)}{\left|x-x_{i}^{k}\right|^{(n+1 / 2) p}} d x \\
\leq & C 2^{k p}\left(d_{i}^{k}\right)^{(n+1 / 2) p}\left(\left(\frac{3}{2}\right)^{\left(k-k_{0}\right) /(n+\beta)} \sqrt{n} d_{i}^{k}\right)^{-(n+1 / 2) p} \\
& \times w\left(\widetilde{B_{i}^{k}}\right) .
\end{aligned}
$$

Since $w \in A_{(1+1 / 2 n) p}$, then there exists a $\epsilon>0$ such that $w \epsilon$ $A_{(1+1 / 2 n) p-\epsilon}$. Thus, we have

$$
w\left(\widetilde{B_{i}^{k}}\right) \leq\left(\frac{3}{2}\right)^{\left(\left(k-k_{0}\right) /(n+\beta)\right)((n+1 / 2) p-\epsilon)} w\left(B_{i}^{k}\right) .
$$

Hence,

$$
I_{1} \leq C 2^{k p}\left(\frac{3}{2}\right)^{-\left(\left(k-k_{0}\right) /(n+\beta)\right) n \epsilon} w\left(B_{i}^{k}\right) .
$$

For $n /(n+\beta)<p \leq 1$ and $w \in A_{q}$ with $q=(1+\beta / n) p>1$, we can see that $\left[n\left(q_{w} / p-1\right)\right]=0$. Since $t \geq\left|x-x_{i}^{k}\right|+\sqrt{n} d_{i}^{k}$, we have $B_{i}^{k} \subset\{y:|x-y| \leq t\}$. By the vanishing moment condition of $b_{i}^{k}$,

$$
\begin{aligned}
& \int_{\left(\widetilde{B_{i}^{k}}\right)^{c}}\left(\int_{\left|x-x_{i}^{k}\right|+\sqrt{n} d_{i}^{k}} \mid \int_{B_{i}^{k}}\left(\frac{\Omega(x-y)}{|x-y|^{n-1}}-\frac{\Omega\left(x-x_{i}^{k}\right)}{\left|x-x_{i}^{k}\right|^{n-1}}\right)\right. \\
& \left.\times\left. b_{i}^{k}(y) d y\right|^{2} \frac{d t}{t^{3}}\right)^{p / 2} w(x) d x .
\end{aligned}
$$


But

$$
\begin{aligned}
& \left|\int_{B_{i}^{k}}\left(\frac{\Omega(x-y)}{|x-y|^{n-1}}-\frac{\Omega\left(x-x_{i}^{k}\right)}{\left|x-x_{i}^{k}\right|^{n-1}}\right) b_{i}^{k}(y) d y\right| \\
& \quad \leq \int_{B_{i}^{k}}\left|\frac{1}{|x-y|^{n-1}}-\frac{1}{\left|x-x_{i}^{k}\right|^{n-1}}\right|\left|\Omega(x-y) b_{i}^{k}(y)\right| d y \\
& \quad+\int_{B_{i}^{k}} \frac{\left|\Omega(x-y)-\Omega\left(x-x_{i}^{k}\right)\right|}{\left|x-x_{i}^{k}\right|^{n-1}}\left|b_{i}^{k}(y)\right| d y \\
& \quad=K_{1}+K_{2} .
\end{aligned}
$$

Note that for any $y \in B_{i}^{k}$ and $x \in\left(\widetilde{B_{i}^{k}}\right)^{c},|x-y| \approx\left|x-x_{i}^{k}\right|$. Thus,

$$
\begin{aligned}
K_{1} & \leq C\|\Omega\|_{L^{\infty}}\left\|b_{i}^{k}\right\|_{L^{\infty}} \int_{B_{i}^{k}}\left|\frac{1}{|x-y|^{n-1}}-\frac{1}{\left|x-x_{i}^{k}\right|^{n-1}}\right| d y \\
& \leq C\left\|b_{i}^{k}\right\|_{L^{\infty}} \frac{\left(d_{i}^{k}\right)^{n+1}}{\left|x-x_{i}^{k}\right|^{n}} .
\end{aligned}
$$

From the fact that $\Omega \in \operatorname{Lip}_{\alpha}\left(S^{n-1}\right)$ and $\Omega$ is homogeneous of degree zero, we get

$$
\begin{aligned}
& \left|\Omega(x-y)-\Omega\left(x-x_{i}^{k}\right)\right| \\
& \quad \leq\left|\Omega\left(\frac{x-y}{|x-y|}\right)-\Omega\left(\frac{x-x_{i}^{k}}{\left|x-x_{i}^{k}\right|}\right)\right| \\
& \quad \leq C\left|\frac{x-y}{|x-y|}-\frac{x-x_{i}^{k}}{\left|x-x_{i}^{k}\right|}\right|^{\alpha} \\
& \quad \leq C\left(\frac{\left|y-x_{i}^{k}\right|}{\left|x-x_{i}^{k}\right|}\right)^{\alpha} .
\end{aligned}
$$

Then,

$$
K_{2} \leq C\left\|b_{i}^{k}\right\|_{L^{\infty}} \frac{\left(d_{i}^{k}\right)^{n+\alpha}}{\left|x-x_{i}^{k}\right|^{n-1+\alpha}} .
$$

For $x \in\left(\widetilde{B_{i}^{k}}\right)^{c}$, we have $\left|x-x_{i}^{k}\right| \approx\left|x-x_{i}^{k}\right|+\sqrt{n} d_{i}^{k}$. Then,

$$
\int_{\left|x-x_{i}^{k}\right|+\sqrt{n} d_{i}^{k}}^{\infty} \frac{d t}{t^{3}} \leq \frac{C}{\left|x-x_{i}^{k}\right|^{2}} .
$$

Hence,

$$
\begin{aligned}
I_{2} \leq & C 2^{k p}\left(d_{i}^{k}\right)^{(n+1) p} \int_{\left(\widetilde{B_{i}^{k}}\right)^{c}} \frac{w(x)}{\left|x-x_{i}^{k}\right|^{(n+1) p}} d x \\
& +C 2^{k p}\left(d_{i}^{k}\right)^{(n+\alpha) p} \int_{\left(\widetilde{B_{i}^{k}}\right)^{c}} \frac{w(x)}{\left|x-x_{i}^{k}\right|^{(n+\alpha) p}} d x \\
= & L_{1}+L_{2} .
\end{aligned}
$$

Since $w \in A_{p(1+\beta / n)}$ and $\beta=\min \{\alpha, 1 / 2\}$, then $w \epsilon$ $A_{p(1+1 / n)}$ and $w \in A_{p(1+1 / n)-\epsilon}$ for some $\epsilon>0$. From Lemma 6,

$$
\begin{aligned}
L_{1} & \leq C 2^{k p}\left(\frac{3}{2}\right)^{-\left(\left(k-k_{0}\right) /(n+\beta)\right) p(n+1)} w\left(\widetilde{B_{i}^{k}}\right) \\
& \leq C 2^{k p}\left(\frac{3}{2}\right)^{-\left(\left(k-k_{0}\right) /(n+\beta)\right) n \epsilon} w\left(B_{i}^{k}\right) .
\end{aligned}
$$

Analogously, we have

$$
L_{2} \leq C 2^{k p}\left(\frac{3}{2}\right)^{-\left(\left(k-k_{0}\right) /(n+\beta)\right) n \epsilon} w\left(B_{i}^{k}\right) .
$$

Then,

$$
I_{2} \leq C 2^{k p}\left(\frac{3}{2}\right)^{-\left(\left(k-k_{0}\right) /(n+\beta)\right) n \epsilon} w\left(B_{i}^{k}\right) .
$$

Hence,

$$
\begin{aligned}
M_{22} & \leq C \sum_{k=k_{0}+1}^{\infty} \sum_{i} 2^{k p}\left(\frac{3}{2}\right)^{-\left(\left(k-k_{0}\right) /(n+\beta)\right) n \epsilon} w\left(B_{i}^{k}\right) \\
& \leq C\|f\|_{\mathrm{WH}_{w}^{p}}^{p} \sum_{k=k_{0}+1}^{\infty}\left(\frac{3}{2}\right)^{-\left(\left(k-k_{0}\right) /(n+\beta)\right) n \epsilon} \\
& \leq C\|f\|_{\mathrm{WH}_{w}^{p}}^{p}
\end{aligned}
$$

Combining the estimate of $M_{21}$ and $M_{22}$, we get $M_{2} \leq$ $C\|f\|_{\mathrm{WH}_{w}^{p}}^{p}$. This completes the proof of the Theorem 1 .

\section{Proof of Theorem 2}

We follow the strategy of the proof of Theorem 1. For any given $\lambda>0$, we may choose $k_{0} \in \mathbb{Z}$ such that $2^{k_{0}} \leq \lambda<2^{k_{0}+1}$. For every $f \in \mathrm{WH}_{w}^{1}$, and then by Lemma 8 , we can write

$$
f=\sum_{k=-\infty}^{\infty} f_{k}=\sum_{k=-\infty}^{k_{0}} f_{k}+\sum_{k=k_{0}+2}^{\infty} f_{k}=F_{1}+F_{2}
$$

where $F_{1}=\sum_{k=-\infty}^{k_{0}} f_{k}=\sum_{k=-\infty}^{k_{0}} \sum_{i} b_{i}^{k}, F_{2}=\sum_{k=k_{0}+2}^{\infty} f_{k}=$ $\sum_{k=k_{0}+2}^{\infty} \sum_{i} b_{i}^{k}$, and $\left\{b_{i}^{k}\right\}$ satisfies (a)-(c) in Lemma 8. Then,

$$
\begin{aligned}
& \lambda w\left(\left\{x \in R^{n}:\left|\mu_{\Omega}(f)(x)\right|>\lambda\right\}\right) \\
& \leq \lambda w\left(\left\{x \in R^{n}:\left|\mu_{\Omega}\left(F_{1}\right)(x)\right|>\frac{\lambda}{2}\right\}\right) \\
& \quad+\lambda w\left(\left\{x \in R^{n}:\left|\mu_{\Omega}\left(F_{2}\right)(x)\right|>\frac{\lambda}{2}\right\}\right)=P_{1}+P_{2} .
\end{aligned}
$$

Similar to the estimate of $M_{1}$ in the proof of Theorem 1, we obtain

$$
P_{1} \leq C\|f\|_{\mathrm{WH}_{w}^{1}}
$$


We set

$$
A_{k_{0}}=\bigcup_{k=k_{0}+1}^{\infty} \bigcup_{i} \widetilde{B_{i}^{k}}
$$

where $\widetilde{B_{i}^{k}}=B\left(x_{i}^{k},(3 / 2)^{\left(k-k_{0}\right) / n} \sqrt{n} d_{i}^{k}\right)$. Then, we get

$$
\begin{aligned}
P_{2} \leq & \lambda w\left(\left\{x \in A_{k_{0}}:\left|\mu_{\Omega}\left(F_{2}\right)(x)\right|>\frac{\lambda}{2}\right\}\right) \\
& +\lambda w\left(\left\{x \in\left(A_{k_{0}}\right)^{c}:\left|\mu_{\Omega}\left(F_{2}\right)(x)\right|>\frac{\lambda}{2}\right\}\right) \\
= & P_{21}+P_{22} .
\end{aligned}
$$

Since $w \in A_{1}$, and then by Lemmas 5 and 8 and the fact $2^{k_{0}} \leq$ $\lambda<2^{k_{0}+1}$, similar to the estimate in the proof of Theorem 1 , we get

$$
P_{21} \leq C\|f\|_{\mathrm{WH}_{w}^{1}}
$$

An application of Chebyshev's inequality gives us that

$$
\begin{aligned}
P_{22} & \leq C \int_{\left(A_{k_{0}}\right)^{c}}\left|\mu_{\Omega}\left(F_{2}\right)\right| w(x) d x \\
& \leq C \sum_{k=k_{0}+2}^{\infty} \sum_{i} \int_{\left(\widetilde{B}_{i}^{k}\right)^{c}}\left|\mu_{\Omega}\left(b_{i}^{k}\right)(x)\right| w(x) d x .
\end{aligned}
$$

Below, we give the estimate of integral $J=$ $\int_{\left(\widetilde{B_{i}^{k}}\right)^{c}}\left|\mu_{\Omega}\left(b_{i}^{k}\right)(x)\right| w(x) d x$. We have

$$
\begin{aligned}
J \leq & \int_{\left(\widetilde{B}_{i}^{k}\right)^{c}}\left(\int_{0}^{\left|x-x_{i}^{k}\right|+\sqrt{n} d_{i}^{k}}\left|\int_{|x-y| \leq t} \frac{\Omega(x-y)}{|x-y|^{n-1}} b_{i}^{k}(y) d y\right|^{2} \frac{d t}{t^{3}}\right)^{1 / 2} \\
& \times w(x) d x \\
+ & \int_{\left(\widetilde{B}_{i}^{k}\right)^{c}}\left(\int_{\left|x-x_{i}^{k}\right|+\sqrt{n} d_{i}^{k}}^{\infty}\left|\int_{|x-y| \leq t} \frac{\Omega(x-y)}{|x-y|^{n-1}} b_{i}^{k}(y) d y\right|^{2} \frac{d t}{t^{3}}\right)^{1 / 2} \\
& \times w(x) d x \\
= & J_{1}+J_{2} .
\end{aligned}
$$

For $y \in B_{i}^{k}$ and $x \in\left(\widetilde{B_{i}^{k}}\right)^{c}$, we have $|x-y| \approx\left|x-x_{i}^{k}\right| \approx$ $\left|x-x_{i}^{k}\right|+\sqrt{n} d_{i}^{k}$. Thus,

$$
\left|\frac{1}{|x-y|^{2}}-\frac{1}{\left(\left|x-x_{i}^{k}\right|+\sqrt{n} d_{i}^{k}\right)^{2}}\right| \leq \frac{C d_{i}^{k}}{|x-y|^{3}} .
$$

Then, Minkowski's inequality for integral gives us that

$$
\begin{aligned}
J_{1} \leq \int_{\left({\widetilde{B_{i}^{k}}}^{c}\right.} \int_{B_{i}^{k}} \frac{|\Omega(x-y)|}{|x-y|^{n-1}}\left|b_{i}^{k}(y)\right| \\
\times\left(\int_{|x-y|}^{\left|x-x_{i}^{k}\right|+\sqrt{n} d_{i}^{k}} \frac{d t}{t^{3}}\right)^{1 / 2} d y w(x) d x \\
\leq C\left(d_{i}^{k}\right)^{1 / 2} \int_{B_{i}^{k}}\left(\int_{\left.\widetilde{B}_{i}^{k}\right)}^{c} \frac{|\Omega(x-y)|}{|x-y|^{n+1 / 2}} w(x) d x\right) \\
\times\left|b_{i}^{k}(y)\right| d y .
\end{aligned}
$$

Since $w^{q^{\prime}} \in A_{1}$, then $w \in A_{1} \subset A_{1+1 / 2 n}$. From Lemmas 6 and 5 , we have

$$
\begin{aligned}
& \left(\int_{\left(\widetilde{B_{i}^{k}}\right)^{c}} \frac{w(x)^{q^{\prime}}}{|x-y|^{n+1 / 2}} d x\right)^{1 / q^{\prime}} \\
& \leq C\left(\left(\frac{3}{2}\right)^{\left(k-k_{0}\right) / n} d_{i}^{k}\right)^{-n / q^{\prime}-1 / 2 q^{\prime}}\left(w^{q^{\prime}}\left(\widetilde{B_{i}^{k}}\right)\right)^{1 / q^{\prime}} \\
& \leq C\left(\left(\frac{3}{2}\right)^{\left(k-k_{0}\right) / n} d_{i}^{k}\right)^{-n / q^{\prime}-1 / 2 q^{\prime}} \\
& \quad \times\left(\left(\frac{3}{2}\right)^{\left(k-k_{0}\right) / n}\right)^{n / q^{\prime}}\left(w^{q^{\prime}}\left(B_{i}^{k}\right)\right)^{1 / q^{\prime}} \\
& \leq C\left(\left(\frac{3}{2}\right)^{\left(k-k_{0}\right) / n} d_{i}^{k}\right)^{-1 / 2 q^{\prime}} \inf _{x \in B_{i}^{k}} w(x) .
\end{aligned}
$$

Hence, for $y \in B_{i}^{k}$, Hölder's inequality and $\Omega \in L^{q}\left(S^{n-1}\right)$ imply that

$$
\int_{\left({\widetilde{B_{i}^{k}}}^{c}\right.} \frac{|\Omega(x-y)|}{|x-y|^{n+1 / 2}} w(x) d x
$$

$$
\begin{aligned}
& \leq\left(\int_{\left(\widetilde{B_{i}^{k}}\right)^{c}} \frac{|\Omega(x-y)|^{q}}{|x-y|^{n+1 / 2}} d x\right)^{1 / q} \\
& \quad \times\left(\int_{\left(\widetilde{B}_{i}^{k}\right)} \frac{w(x)^{q^{\prime}}}{|x-y|^{n+1 / 2}} d x\right)^{1 / q^{\prime}} \\
& \leq\left(\int_{(3 / 2)^{\left(k-k_{0}\right) / n} \sqrt{n} d_{i}^{k}}^{\infty} \int_{S^{n-1}} \frac{\left|\Omega\left(x^{\prime}\right)\right|^{q}}{r^{n+1 / 2}} r^{n-1} d \sigma\left(x^{\prime}\right) d r\right)^{1 / q}
\end{aligned}
$$

$$
\begin{aligned}
& \times\left(\left(\frac{3}{2}\right)^{\left(k-k_{0}\right) / n} d_{i}^{k}\right)^{-1 / 2 q^{\prime}} \inf _{x \in B_{i}^{k}} w(x) \\
\leq & C\left(\left(\frac{3}{2}\right)^{\left(k-k_{0}\right) / n} d_{i}^{k}\right)^{-1 / 2 q}
\end{aligned}
$$




$$
\begin{aligned}
& \times\left(\left(\frac{3}{2}\right)^{-\left(k-k_{0}\right) / n} d_{i}^{k}\right)^{-1 / 2 q^{\prime}} \inf _{x \in B_{i}^{k}} w(x) \\
\leq & C\left(\left(\frac{3}{2}\right)^{\left(k-k_{0}\right) / n} d_{i}^{k}\right)^{-1 / 2} \inf _{x \in B_{i}^{k}} w(x) .
\end{aligned}
$$

Then,

$$
\begin{aligned}
J_{1} & \leq C 2^{k}\left(d_{i}^{k}\right)^{1 / 2}\left(\left(\frac{3}{2}\right)^{\left(k-k_{0}\right) / n} d_{i}^{k}\right)^{-1 / 2} \inf _{x \in B_{i}^{k}} w(x)\left|B_{i}^{k}\right| \\
& \leq C 2^{k} w\left(B_{i}^{k}\right)\left(\frac{2}{3}\right)^{\left(k-k_{0}\right) / 2 n} .
\end{aligned}
$$

We now estimate $J_{2}$. Since $t \geq\left|x-x_{i}^{k}\right|+\sqrt{n} d_{i}^{k}$, we have $B_{i}^{k} \subset\{y:|x-y| \leq t\}$ and hence

$J_{2}$

$$
\begin{aligned}
=\int_{\left({\widetilde{B_{i}^{k}}}^{c}\right.}\left(\int_{\left|x-x_{i}^{k}\right|+\sqrt{n} d_{i}^{k}}^{\infty} \mid \int_{B_{i}^{k}}\left(\frac{\Omega(x-y)}{|x-y|^{n-1}}\right.\right. \\
\\
\left.\left.-\frac{\Omega\left(x-x_{i}^{k}\right)}{\left|x-x_{i}^{k}\right|^{n-1}}\right)\left.b_{i}^{k}(y) d y\right|^{2} \frac{d t}{t^{3}}\right)^{1 / 2}
\end{aligned}
$$$$
\times w(x) d x .
$$

Denote $R_{j}^{k}=2^{j}(3 / 2)^{\left(k-k_{0}\right) / n} \sqrt{n}$. Minkowski's inequality for integrals and the fact $\left|x-x_{i}^{k}\right|+\sqrt{n} d_{i}^{k} \approx\left|x-x_{i}^{k}\right|$ for $x \in\left(\widetilde{B_{i}^{k}}\right)^{c}$ give us

$$
\begin{aligned}
J_{2} \leq \int_{\left(\widetilde{B}_{i}^{k}\right)^{c}} \int_{B_{i}^{k}}\left|\frac{\Omega(x-y)}{|x-y|^{n-1}}-\frac{\Omega\left(x-x_{i}^{k}\right)}{\left|x-x_{i}^{k}\right|^{n-1}}\right|\left|b_{i}^{k}(y)\right| \\
\quad \times\left(\int_{\left|x-x_{i}^{k}\right|+\sqrt{n} d_{i}^{k}}^{\infty} \frac{d t}{t^{3}}\right)^{1 / 2} d y w(x) d x \\
\leq \int_{\left(\widetilde{B}_{i}^{k}\right)^{c}} \int_{B_{i}^{k}} \frac{\Omega(x-y)}{|x-y|^{n-1}}-\frac{\Omega\left(x-x_{i}^{k}\right)}{\left|x-x_{i}^{k}\right|^{n-1}} \mid \\
\leq C \int_{B_{i}^{k}} \sum_{j=0}^{\infty} \frac{1}{R_{j}^{k} d_{i}^{k}} \frac{\left|b_{i}^{k}(y)\right|}{\left|x-x_{i}^{k}\right|} d y w(x) d x \\
\quad \times \int_{R_{j}^{k} d_{i}^{k} \leq\left|x-x_{i}^{k}\right| \leq R_{j+1}^{k} d_{i}^{k}}\left|\frac{\Omega(x-y)}{|x-y|^{n-1}}-\frac{\Omega\left(x-x_{i}^{k}\right)}{\left|x-x_{i}^{k}\right|^{n-1}}\right| \\
\times w(x) d x\left|b_{i}^{k}(y)\right| d y .
\end{aligned}
$$

Notice that

$$
\left(w^{q^{\prime}}\left(R_{j+1}^{k} B_{i}^{k}\right)\right)^{1 / q^{\prime}} \leq C\left(R_{j}^{k}\right)^{n / q^{\prime}}\left(d_{i}^{k}\right)^{n / q^{\prime}} \inf _{x \in B_{i}^{k}} w(x) .
$$

By Hölder's inequality and Lemma 7, we have

$$
\begin{aligned}
& \int_{R_{j}^{k} d_{i}^{k} \leq\left|x-x_{i}^{k}\right| \leq R_{j+1}^{k} d_{i}^{k}}\left|\frac{\Omega(x-y)}{|x-y|^{n-1}}-\frac{\Omega\left(x-x_{i}^{k}\right)}{\left|x-x_{i}^{k}\right|^{n-1}}\right| w(x) d x \\
& \leq\left(\int_{R_{j}^{k} d_{i}^{k} \leq\left|x-x_{i}^{k}\right| \leq R_{j+1}^{k} k_{i}^{k}}\left|\frac{\Omega(x-y)}{|x-y|^{n-1}}-\frac{\Omega\left(x-x_{i}^{k}\right)}{\left|x-x_{i}^{k}\right|^{n-1}}\right|^{q} d x\right)^{1 / q} \\
& \quad \times\left(w^{q^{\prime}}\left(R_{j+1}^{k} B_{i}^{k}\right)\right)^{1 / q^{\prime}} \\
& \leq C\left(R_{j}^{k} d_{i}^{k}\right)^{1-n+n / q} \\
& \quad \times\left(\frac{\left|y-x_{i}^{k}\right|}{R_{j}^{k} d_{i}^{k}}+\int_{\left|y-x_{i}^{k}\right| / R_{j+1}^{k} d_{i}^{k} \leq \delta \leq\left|y-x_{i}^{k}\right| / R_{j}^{k} d_{i}^{k}} \frac{\omega_{q}(\delta)}{\delta} d \delta\right) \\
& \quad \times\left(w^{q^{\prime}}\left(R_{j+1}^{k} B_{i}^{k}\right)\right)^{1 / q^{\prime}} \\
& \leq C R_{j}^{k} d_{i}^{k} \inf _{x \in B_{i}^{k}} w(x) \\
& \quad \times\left(\frac{\left|y-x_{i}^{k}\right|}{R_{j}^{k} d_{i}^{k}}+\int_{\left|y-x_{i}^{k}\right| / R_{j+1}^{k} d_{i}^{k} \leq \delta \leq\left|y-x_{i}^{k}\right| / R_{j}^{k} d_{i}^{k}} \frac{\omega_{q}(\delta)}{\delta} d \delta\right) .
\end{aligned}
$$

Then,

$$
\begin{aligned}
J_{2} \leq & C 2^{k} \inf _{x \in B_{i}^{k}} w(x)\left|B_{i}^{k}\right| \\
\times & \sum_{j=0}^{\infty}\left(\frac{1}{2^{j}}\left(\frac{2}{3}\right)^{\left(k-k_{0}\right) / n}\right. \\
& \left.\quad+\int_{\left|y-x_{i}^{k}\right| / R_{j+1}^{k} d_{i}^{k} \leq \delta \leq\left|y-x_{i}^{k}\right| / R_{j}^{k} d_{i}^{k}} \frac{\omega_{q}(\delta)}{\delta} d \delta\right) \\
\leq & C 2^{k} w\left(B_{i}^{k}\right)\left(\left(\frac{2}{3}\right)^{\left(k-k_{0}\right) / n}+\int_{0}^{(2 / 3)^{\left(k-k_{0}\right) / n}} \frac{\omega_{q}(\delta)}{\delta} d \delta\right) .
\end{aligned}
$$

But

$$
\begin{aligned}
& \int_{0}^{(2 / 3)^{\left(k-k_{0}\right) / n}} \frac{\omega_{q}(\delta)}{\delta} d \delta \\
& \quad \leq \frac{C}{\left[1+\left(k-k_{0}\right) \log (3 / 2)\right]^{\sigma}} \int_{0}^{1} \frac{w_{q}(\delta)}{\delta}(1+|\log \delta|)^{\sigma} d \delta \\
& \quad<\frac{C}{\left(k-k_{0}\right)^{\sigma}},
\end{aligned}
$$


from which we get

$$
J_{2} \leq C 2^{k} w\left(B_{i}^{k}\right)\left(\left(\frac{2}{3}\right)^{\left(k-k_{0}\right) / n}+\frac{C}{\left(k-k_{0}\right)^{\sigma}}\right) .
$$

Thus,

$$
\begin{aligned}
P_{22} & \leq C \sum_{k=k_{0}+2}^{\infty} \sum_{i} \int_{\left(\widetilde{B_{i}^{k}}\right)^{c}}\left|\mu_{\Omega}\left(b_{i}^{k}\right)(x)\right| w(x) d x \\
& \leq C \sum_{k=k_{0}+2}^{\infty} 2^{k} \sum_{i} w\left(B_{i}^{k}\right)\left(\left(\frac{2}{3}\right)^{\left(k-k_{0}\right) / n}+\frac{C}{\left(k-k_{0}\right)^{\sigma}}\right) \\
& \leq C\|f\|_{\mathrm{WH}_{w}^{1}} \sum_{k=k_{0}+2}^{\infty}\left(\left(\frac{2}{3}\right)^{\left(k-k_{0}\right) / n}+\frac{C}{\left(k-k_{0}\right)^{\sigma}}\right) \\
& \leq C\|f\|_{\mathrm{WH}_{w}^{1}} .
\end{aligned}
$$

This completes the proof of Theorem 2.

\section{Conflict of Interests}

The authors declare that there is no conflict of interests regarding the publication of this paper.

\section{References}

[1] L. Hörmander, "Estimates for translation invariant operators in $L^{p}$ spaces," Acta Mathematica, vol. 104, pp. 93-140, 1960.

[2] E. M. Stein, "On the functions of Littlewood-Paley, Lusin, and Marcinkiewicz," Transactions of the American Mathematical Society, vol. 88, pp. 430-466, 1958.

[3] A. Benedek, A. P. Calderón, and R. Panzone, "Convolution operators on Banach space valued functions," Proceedings of the National Academy of Sciences of the United States of America, vol. 48, pp. 356-365, 1962.

[4] A. Torchinsky and S. L. Wang, "A note on the Marcinkiewicz integral," Colloquium Mathematicum, vol. 60-61, no. 1, pp. 235243, 1990.

[5] Y. Ding, D. Fan, and Y. Pan, "Weighted boundedness for a class of rough Marcinkiewicz integrals," Indiana University Mathematics Journal, vol. 48, no. 3, pp. 1037-1055, 1999.

[6] Y. Ding, M. Y. Lee, and C. C. Lin, "Marcinkiewicz integral on weighted Hardy spaces," Archiv der Mathematik, vol. 80, no. 6, pp. 620-629, 2003.

[7] C. C. Lin and Y. C. Lin, " $H_{w}^{p}-L_{w}^{p}$ boundedness of Marcinkiewicz integral," Integral Equations and Operator Theory, vol. 58, no. 1, pp. 87-98, 2007.

[8] Y. Ding, S. Lu, and Q. Xue, "Marcinkiewicz integral on Hardy spaces," Integral Equations and Operator Theory, vol. 42, no. 2, pp. 174-182, 2002.

[9] B. Muckenhoupt, "Weighted norm inequalities for the Hardy maximal function," Transactions of the American Mathematical Society, vol. 165, pp. 207-226, 1972.

[10] J. García-Cuerva and J. L. R. de Francia, Weighted Norm Inequalities and Related Topics, vol. 116 of North-Holland Mathematics Studies, North-Holland, Amsterdam, The Netherlands, 1985.

[11] Y. Ding and S. Lu, "Homogeneous fractional integrals on Hardy spaces," The Tohoku Mathematical Journal, Second Series, vol. 52, no. 1, pp. 153-162, 2000.
[12] C. Fefferman, N. M. Rivière, and Y. Sagher, "Interpolation between $H^{p}$ spaces: the real method," Transactions of the American Mathematical Society, vol. 191, pp. 75-81, 1974.

[13] R. Fefferman and F. Soria, "The space weak $H^{1}$," Studia Mathematica, vol. 85, no. 1, pp. 1-16, 1975.

[14] T. Quek and D. Yang, "Calderón-Zygmund-type operators on weighted weak Hardy spaces over $R^{n}$," Acta Mathematica Sinica, vol. 16, no. 1, pp. 141-160, 2000. 


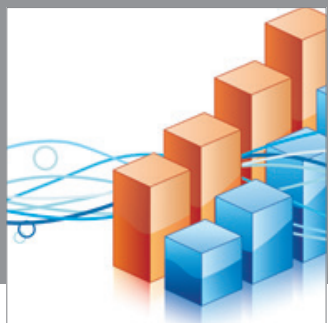

Advances in

Operations Research

mansans

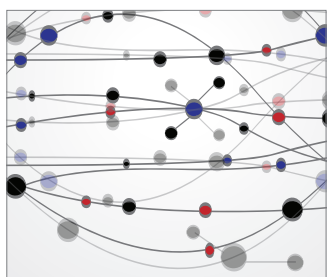

The Scientific World Journal
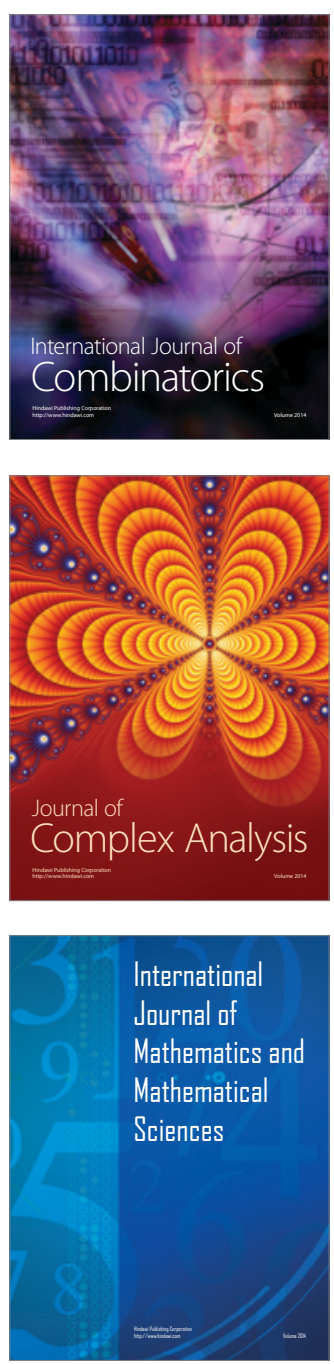
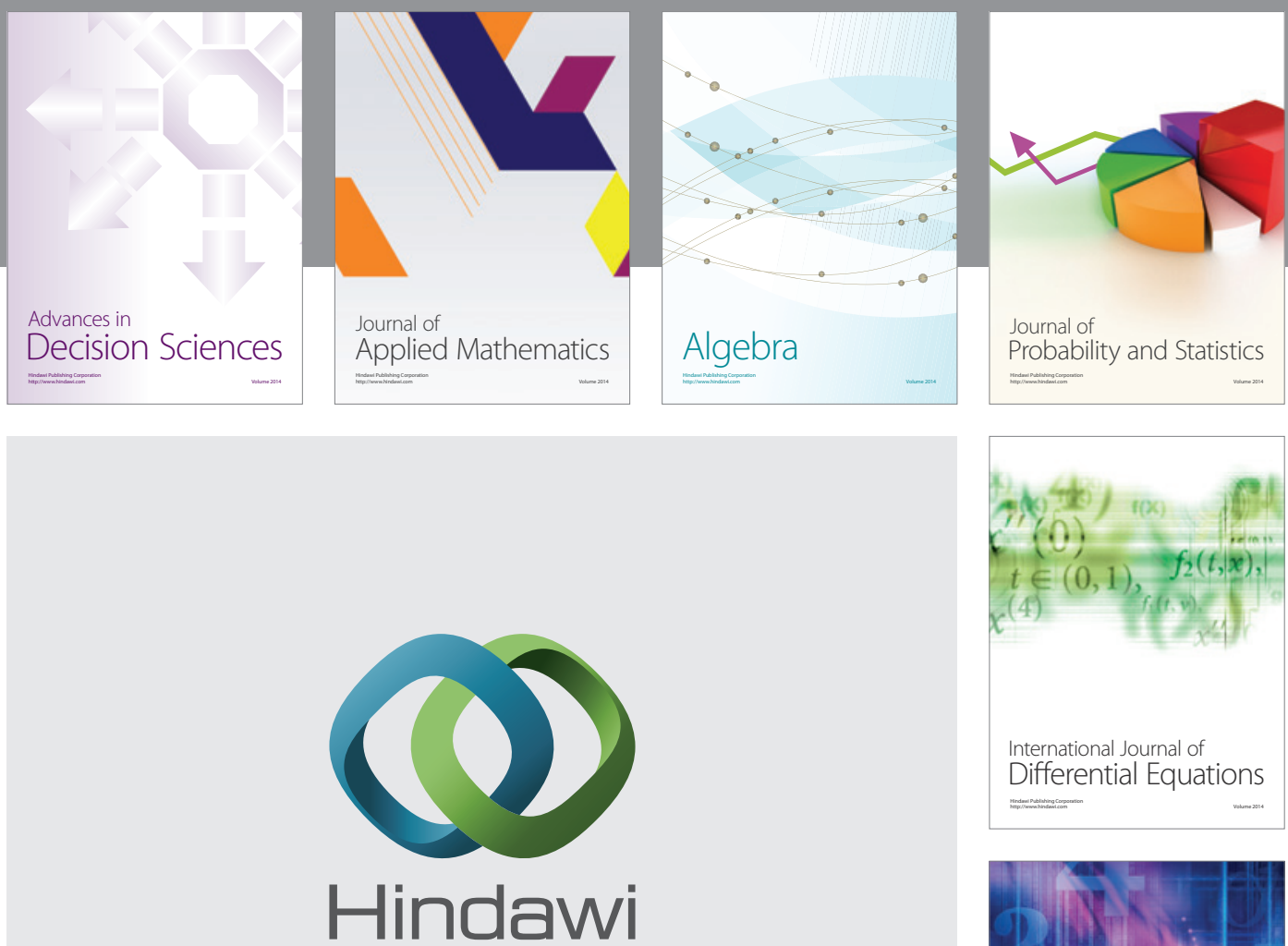

Submit your manuscripts at http://www.hindawi.com
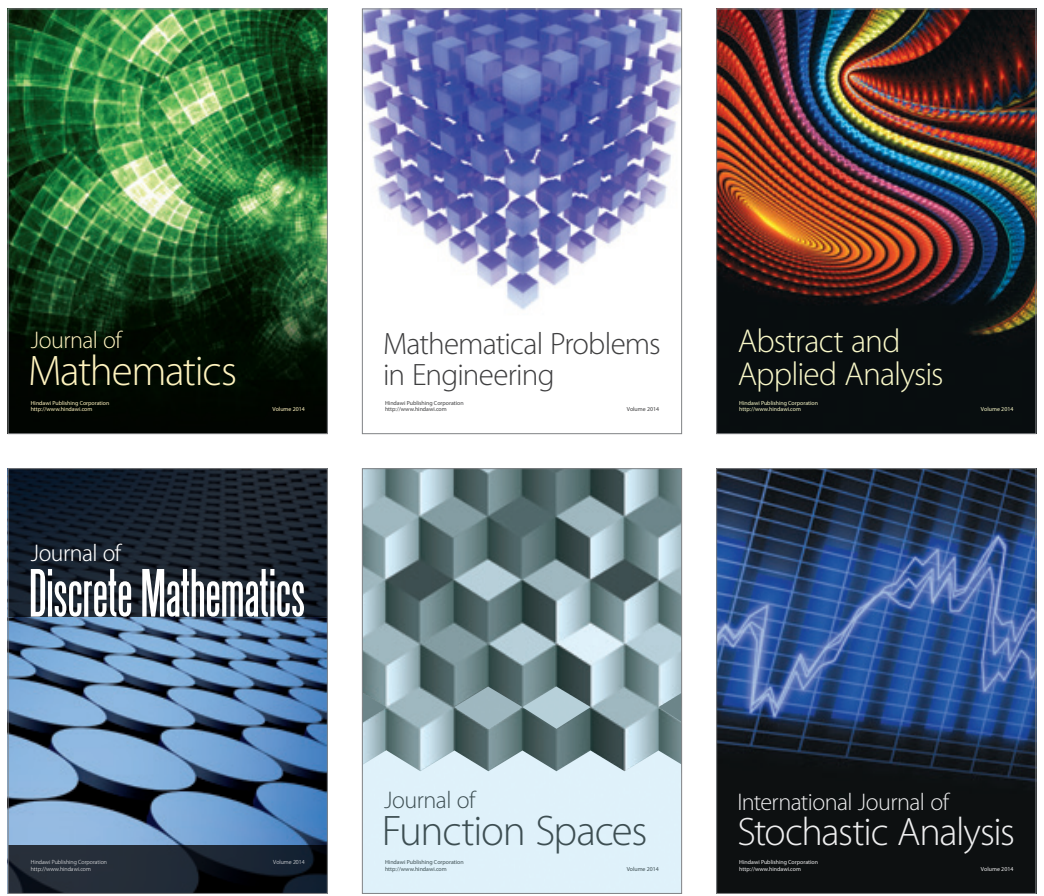

Journal of

Function Spaces

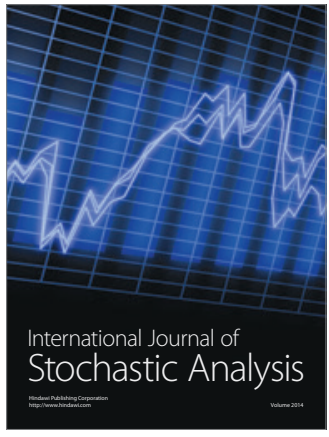

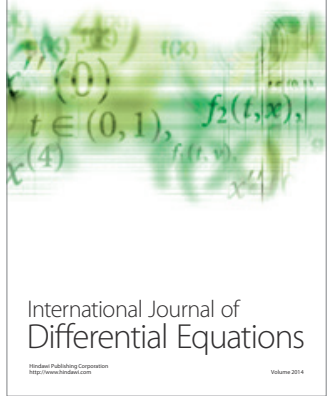
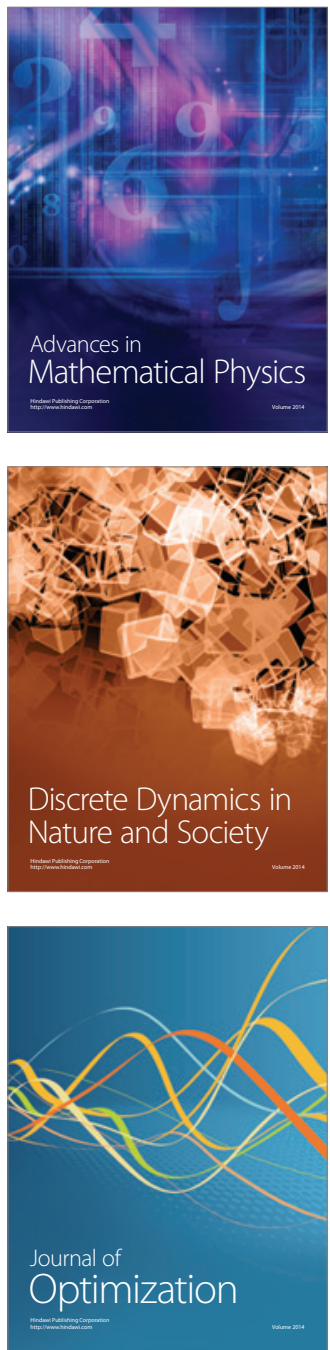